Historic, Archive Document

Do not assume content reflects current scientific knowledge, policies, or practices. 



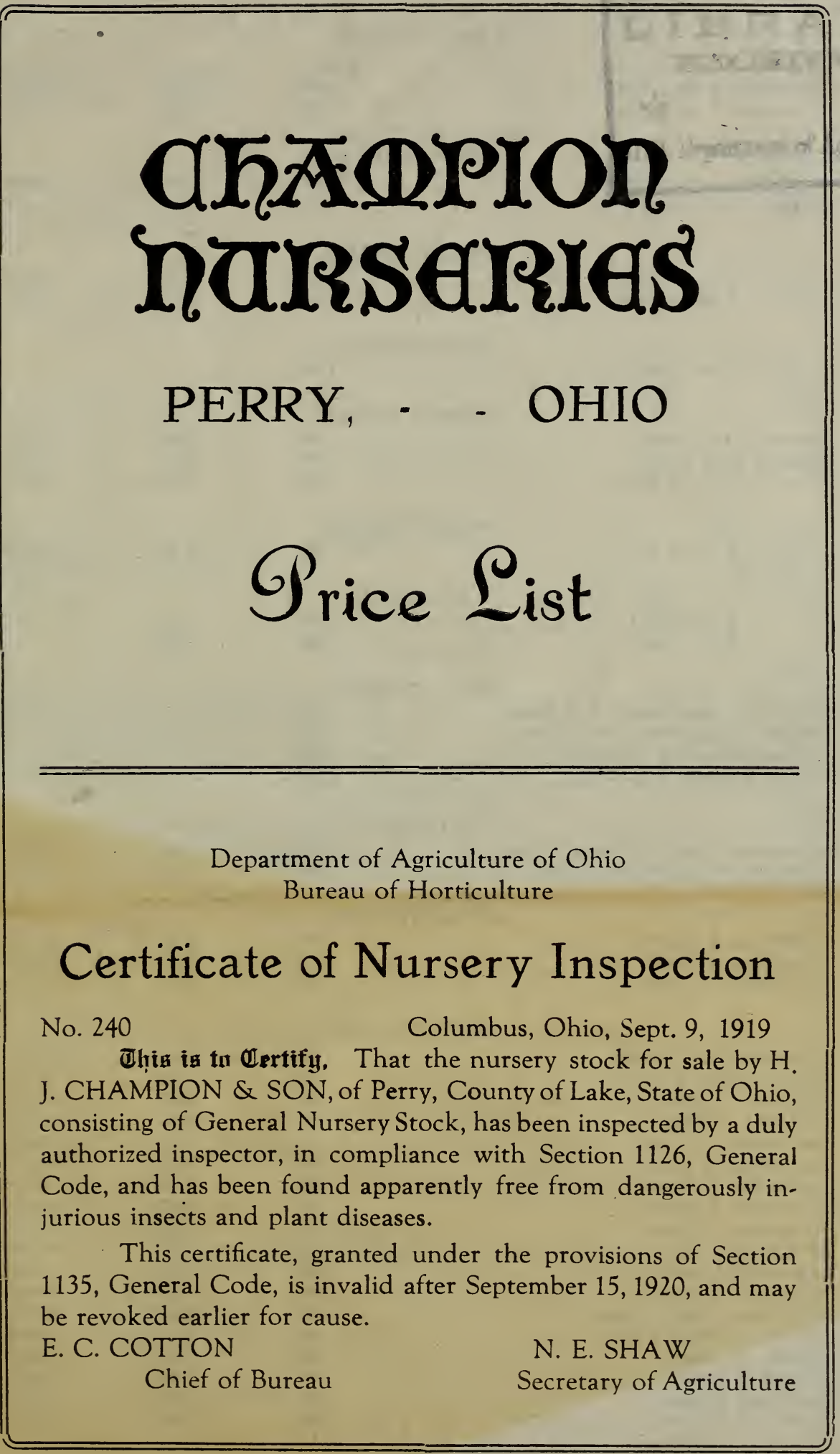


Each

Standard 4 to 5 feet................ 65

\section{PEAR TREES}

Standard 5 to 7 feet................ .90

Dwarf 4 to 5 feet...................90

PEACH TREES

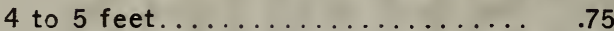

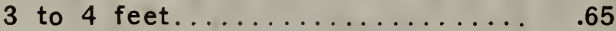

6.50

5.50

55.00

45.00

APRICOTS

4 to 6 feet

1.00

\section{CHERRY TREES}

Sweet 5 to 7 feet................ .95

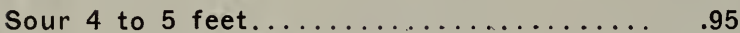

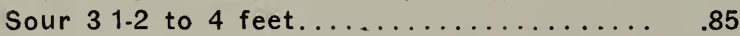

PLUM TREES

5 to 6 feet................ .95

\section{QUINCES}

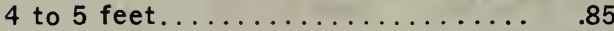

3 to 4 feet................ 80

\section{NUT TREES}

Chestnut Am. Sweet 4 to 5 feet......... .75

Chestnut Spanish 4 to 5 feet........... 1.25

Japan Walnut 6 to 8 feet............. 1.00

Black Walnut 5 to 6 feet............. .95

\section{CURRANTS}

Currents Leading Sorts 2 year.......... .35

3.00

25.00

Currents Perfection 2 year............ $\quad .50$

\section{GOOSEBERRIES}

Downing 2 year................... 40

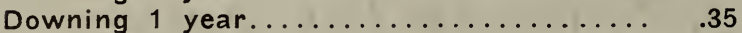

Houghton 2 year.................

\section{GRAPEVINES}

Pocklington 1 year................ .35

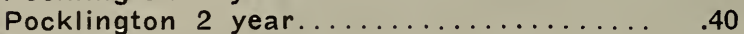

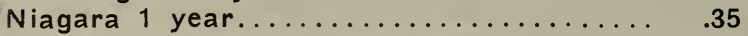

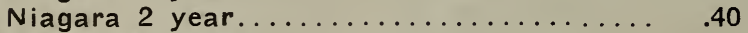

3.00

Delaware 1 year................. 40

30.00

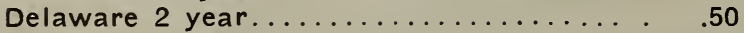

3.50

4.00

4.00

35.00

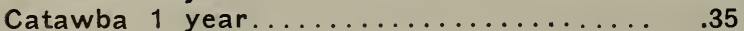

25.00

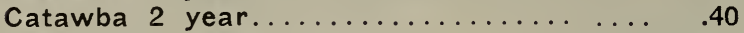

3.50

30.00

3.50

30.00

Brighton 1 year................... .40

4.00

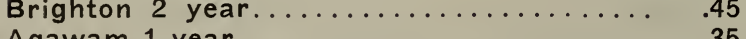

3.00

3.50

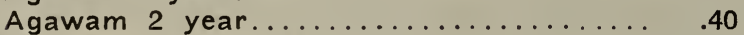

35.00

30.00

35.00

3.00

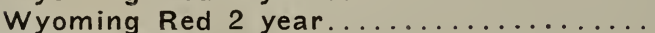




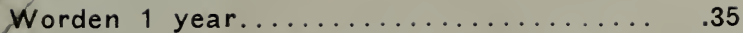

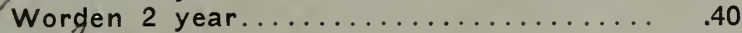

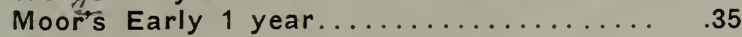

Moor's Early 2 year.

RASPBERRIES BLACK

$$
\text { Per } 10
$$

Kansas, Cumberland, Plum Farmer...... $\quad .75$

Columbian ........................ 1.00

3.50

30.00

Cuthbert, St. Regis.

.75

Per 100
3.50
5.00
4.00

Per 1000

25.00

40.00

35.00

\section{BLACKBERRIES}

Blowers, Eldorado.

Per 10

1.00

Per 100

6.00

STRAWBERRIES

Everbearing

Per 100

3.00

1.50

Per 1000

25.00

Leading Varieties.

1.50

8.50

ASPARAGUS

2 year.

14.00

MULBERRIES

Downing, New American 5 to 6 feet.

Russian 5 to 6 feet.

Rhubarb

Per 10

1.50

1.00 Each

.75 Each

Per 100

10.00

WEEPING TREES

Each

$\$ 1.25$

Wisconsin Willow 6 to 8 feet

1.25

Cut Leaf Weeping Birch 6 to 8 feet.

1.50

Cut Leaf Weeping Birch 8 to 10 feet.

2.00

\section{DECIDUOUS TREES}

Purple Leaved Beech 3 to 4 feet....................... 2.00

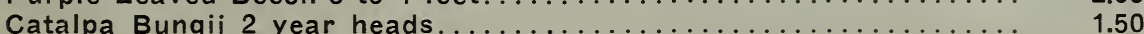

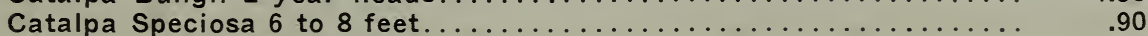

Weirs Cut Leaf Maple 8 to 10 feet....................... 1.50

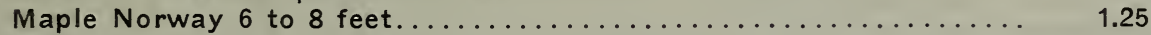

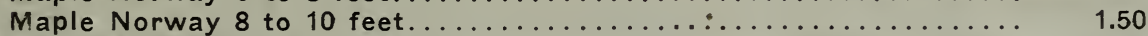

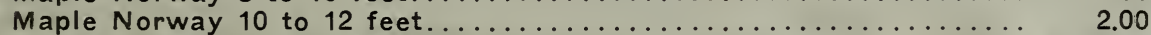

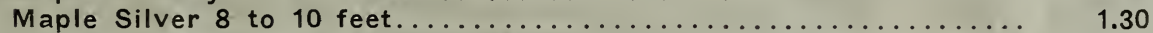

Maple Silver 10 to 12 feet............................. 1.60

European Sycamore 8 to 10 feet........................... 1.25

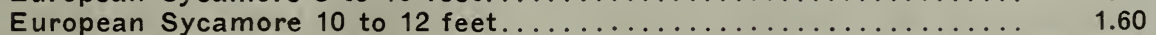

American Elm 8 to 10 feet............................. 1.35

Each

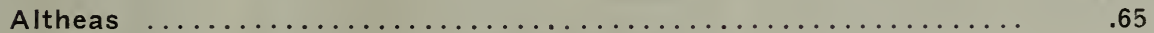

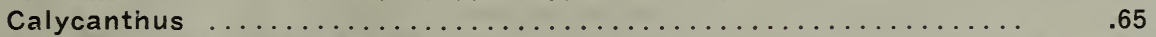

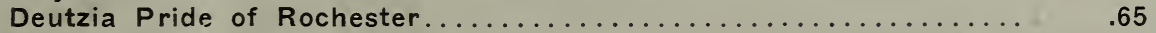

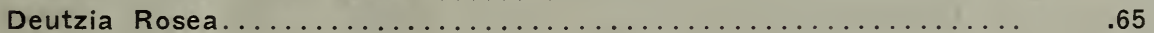

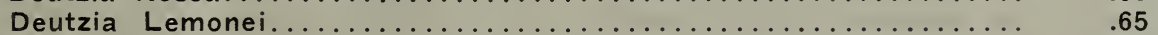

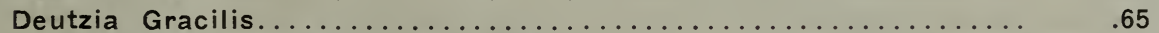

Hydrangea Paniculata Grandiflora . . . . . . . . . . . 50

Hydrangea Arborescens Hills of Snow.................... $\quad .50$

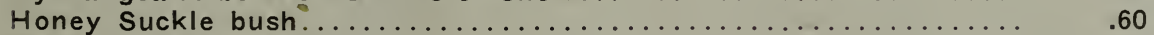

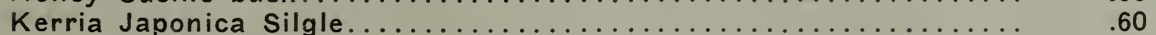

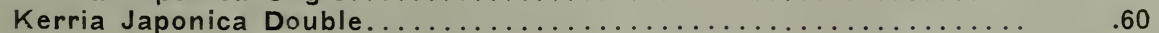

Kerria Japonica Variegated Leaf........................ 60

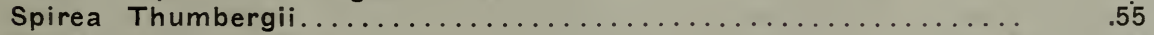

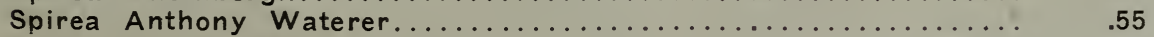

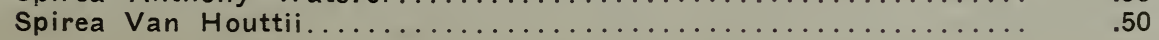




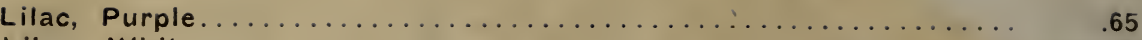

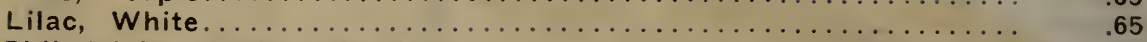

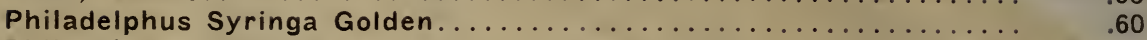

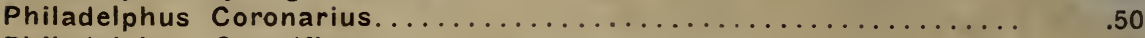

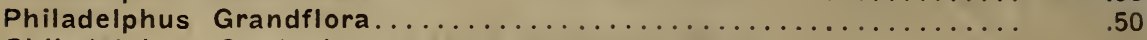

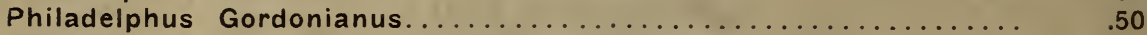

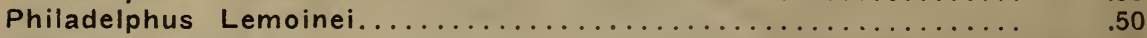

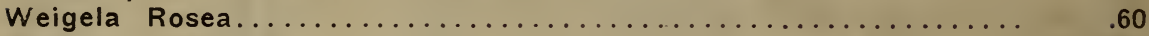

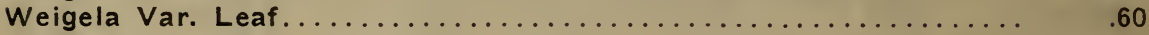

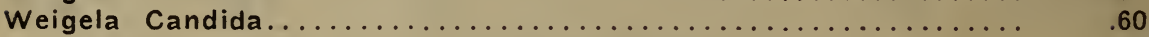

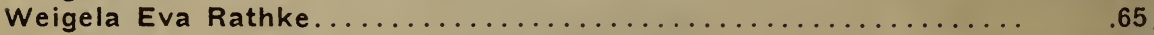

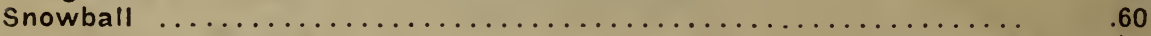

ROSES 75c each.

Eugene Furst, Persian Yellow, Frau Karl Druschki, Anna Diesback. Clio, Marshall P. Wilder, Paul Neyron. Gen. Jack, Ulrich Bruner, Margaret Dickson, Magna Charta, Pink Killarney. White Killarney. Gruss au Teplitz. Mrs. J. H. Lang, White Moss, Crested Moss, Red Moss.

\section{Baby Rambler, Dark Red Baby Rambler. \\ ROSES 80c each}

ROSES $65 \mathrm{c}$ each

Crimson Rambler, White Rambler, Yellow Rambler, Seven Sisters, Dorothy Perkins, Queen of Prarie, Baltimore Bell, Flower of Fairfield, Thousand Beauties, Excelsa, White Dorothy.

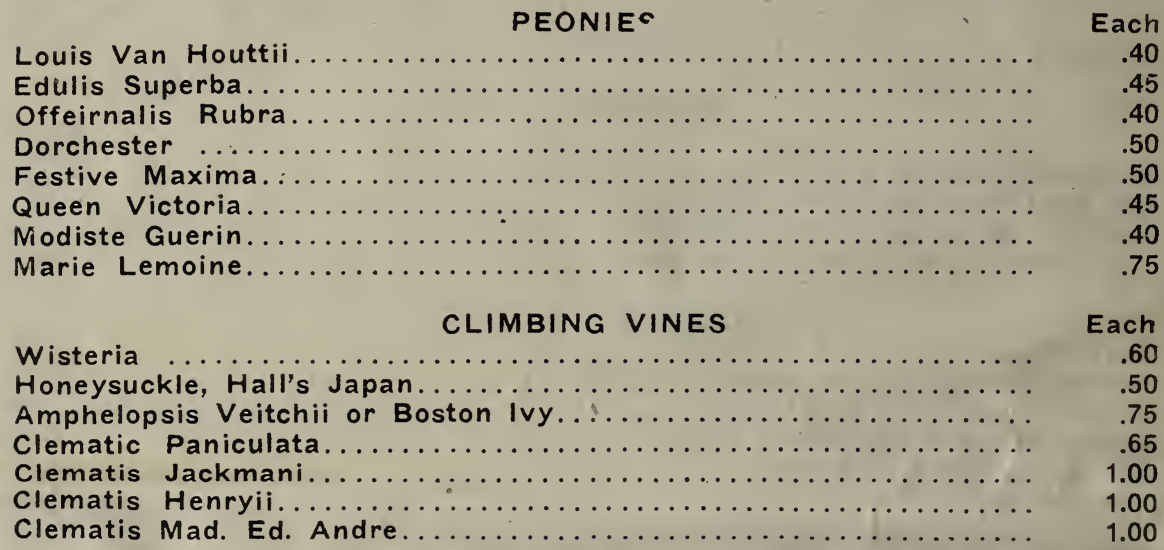

PLANTS FOR HEDGES

California Privet 2 Per 10

California Privet 2 to 3 feet................ \$1.50

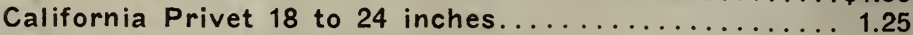

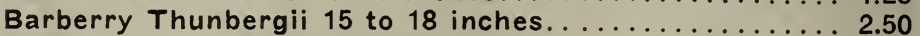

Barberry Thunbergii 18 to 24 inches................ 3.00

Per 100

$\$ 8.00$

7.00

12.00

25.00

\section{EVERGREENS}

Arborvitae American 2 to 3 feet.......................

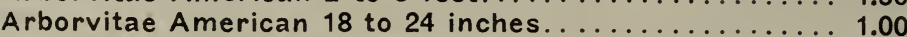

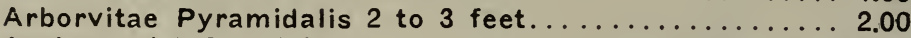

Juniper Irish 3 to 4 feet...................... 2.00

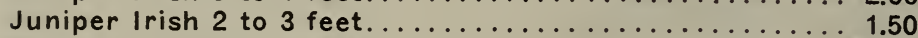

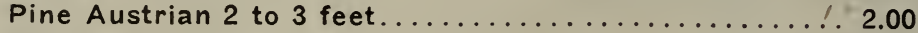

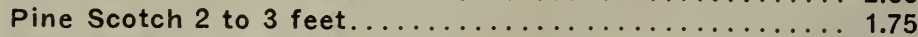

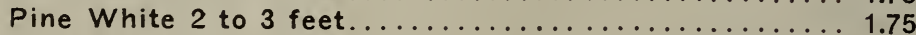

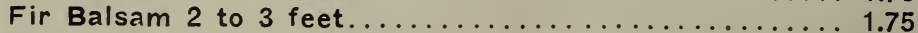

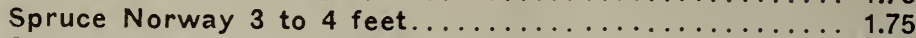

Spruce Norway 2 to 3 feet....................... 1.50

Spruce Norway 11.2 to 3 feet................... 1.00 